\title{
Polynomial instability of evolution operators in Banach spaces
}

\section{Magda LuminiţA RĂmneAnţu, Mihail MEgan and TRAiAn CEAuşu}

\section{ABSTRACT.}

In this paper we consider two concepts of polynomial instability for evolution operators in Banach spaces. Our approach is based on the extension of techniques for exponential instability to the case of polynomial instability. The obtained results are generalizations of some theorems about uniform and nonuniform exponential instability.

\section{DEPARTMENT OF MATHEMATICS}

WEST UNIVERSITY OF TIMIŞOARA

BD. V. PÂRVAN 4, 300223 TIMIŞOARA, ROMANIA

E-mail address: lmramneantu@gmail.com

E-mail address: megan@math.uvt.ro

E-mail address: ceausu@math.uvt.ro

Received: 30.10.2010; In revised form: 09.11.2012; Accepted: 12.12.2012

2010 Mathematics Subject Classification. 34 D05, 34 E05.

Key words and phrases. Evolution operator, polynomial instability.

Corresponding author: Mihail Megan; megan@math.uvt.ro 